\title{
Computation of the changes in gravitational gradient components from GRACE observations and analytical model
}

\author{
Farzam Fatolazadeh $^{1} \cdot$ Behzad Voosoghi $^{1} \cdot$ Mehdi Raoofian Naeeni $^{1}$
}

Received: 15 October 2014/Accepted: 14 November 2015/Published online: 1 December 2015

(C) Akadémiai Kiadó 2015

\begin{abstract}
In this study the gravitational gradient changes caused by faulting on a finite rectangular plane buried in a homogenous half-space are computed using an analytical model which is a function of fault parameters. The sensitivity analysis of the analytical model with optional parameters revealed that the model is sensitive to the most of the fault parameters such as slip, depth, dip and is not sensitive to the strike. Also the results show that the model is sensitive to the length and the width of the fault. Moreover, the gravitation and gravitational gradient changes for the case of the Maule earthquake on 27 February 2010 are directly computed by GRACE observations without need to fault parameters information. Since the high-frequency contents in gravitational field variation can be amplified by deriving the gravitational gradients, the GRACE-derived coseismic gravitational gradient changes clearly delineate the fault lines and better define the extent of the coseismic deformation.
\end{abstract}

Keywords Gravitational gradient changes - Analytical models - Fault parameters · GRACE observations · Maule earthquake

Mehdi Raoofian Naeeni

mraoofian@kntu.ac.ir

Farzam Fatolazadeh

ffatollahzadeh@mail.kntu.ac.ir

Behzad Voosoghi

vosoghi@kntu.ac.ir

1 Faculty of Geodesy and Geomatics Engineering, K. N. Toosi University of Technology,

Tehran, Iran 


\section{Introduction}

Modeling of crustal deformation and exploring the physical impact of this phenomenon is one of the most popular subjects in a ground science particularly in geodesy and geophysics. Numerous studies have been undertaken by many scientists to study co-seismic deformation in a half-space Earth model, a spherical earth model, and even a 3D earth model. For a half-space earth model, Steketee (1958), Maruyama (1964) and Okada (1985), etc. presented analytical expressions for calculating the surface displacement, tilt, and strain resulting from various dislocations. Especially, Okada (1985) summarized previous studies and presented a complete set of analytical formulae for calculating these geodetic deformations. One of the analytical models of computing the gravitational changes due to deformation was presented by Okubo model (Okubo 1991). Okubo (1991) computed gravitational potential changes in a homogenous elastic half space for a point dislocation. He developed his theory to rectangular fault in a half space and computed gravitational potential and gravity changes in the form of fault parameters. Okubo (1992) proposed closed-form expressions to describe potential and gravity changes resulting from dislocations. Because of their mathematical simplicity, these formulations (e.g., Okada 1985; Okubo 1992) have been widely applied to study seismic faults. All of the mathematical models explained above were developed for a deformed earth surface because most traditional gravity measurements are performed on the earth surface. However, advances in modern geodetic techniques, such as GPS, InSAR, altimetry, and GRACE enable better detection of co-seismic deformations such as displacement, gravity change, and strain.

One of the observations resources for comparison to geodynamic models are gravity observations. Earthquake causes redistribution of mass and gravity changes. Some part of this change is due to hydrology and the main part is due to tectonic motion. Changes of gravity field can be detected by observation of the gravity field at different times. Coseismic deformations observed on the Earth surface or modeled by conventional dislocation theory can be compared directly with those observed by gravity satellite missions. Satellite measurements of time-variable gravity field are new data type, capable to modeling and detecting global mass transfers within the Earth. Such a global mass redistribution may lead to significant changes in the Earth's gravity field that is detectable by gravimetric satellites. However, the regional mass transfer such as localized tectonic processes due to earthquake and volcano, can have an indicative influence on gravity fields that may be sensed by GRACE (Ogawa and Heki 2007; Han et al. 2010, 2013; Heki and Matsuo 2010; Linage et al. 2009).

In this study the analytical model of Okubo (1992) is used which computes the gravity changes due to a dislocation on a rectangular fault plane within an elastic, isotropic and homogenous half-space. The formulation of that model is expanded to compute the gravitational gradient changes due to a fault in an elastic and homogenous half space. Also a sensitivity analysis is applied to analytical model to show that the model is sensitive to the fault parameters and is comparable with observations. Moreover, we infer the full gravitational gradient tensor from the GRACE Stokes coefficients by taking the second derivatives of the gravitational potential in a given orthogonal coordinate system without any required information about fault parameters, and apply these quantities to compare with analytical model and discuss the coseismic deformation resulting from the Maule Chilean undersea earthquakes. With the emphasis on the high-frequency components resulting from the second derivatives of the potential, the coseismic gravitational gradient changes delineate more clearly the rupture line, and reveal refined mass redistribution 
features caused by the earthquakes. In addition, some of the gradient components, which are inherently less vulnerable to GRACE stripe errors, help to refine the edges of the mass anomaly.

\section{Data and processing}

\subsection{Gravitational gradient changes using analytical model}

Let's first discuss the displacement field $\vec{u}$ caused by a point dislocation buried in a homogeneous, isotropic and perfectly elastic half-space of density $\rho$. In Fig. 1, a left-hand Cartesian frame is defined in the way that the $x_{1} o x_{2}$ plane spans the surface of the halfspace with $x_{3}$-axis pointing perpendicularly downwards. A point dislocation source is buried at $\left(0,0, \xi_{3}\right)$ in the half-space. The point source can be considered as an infinitesimal fault of area $d \sum$ having the normal direction $\vec{n}=\left(n_{1}, n_{2}, n_{3}\right)$. The dislocation on this infinitesimal fault plane is represented by a vector $\Delta \vec{u}=\left(\Delta u_{1}, \Delta u_{2}, \Delta u_{3}\right)$.

Usually, it is not enough to just use an infinitesimal point dislocation to approximate the effect by a fault plane of finite size. Having described the half space on a point dislocation, let us turn to a more realistic source model with the uniform dislocation on a rectangular fault. How well the gravitation change due to a finite fault can be approximated by using a point dislocation depends on the distance between observation point and the dislocation source, as well as the size of the actual fault. Figure 2 shows a finite rectangular fault plane with its length, width, depth and dip angle of $L, W, d$, and $\delta$ respectively. As indicated by the red arrows in Fig. 2, the dislocation on this fault plane has the along-strike, down dip and tensile components of $U_{1}, U_{2}$ and $U_{3}$ respectively. Under the Cartesian frame $x_{1}, x_{2}, x_{3}$, the dislocation can be represented by a vector $\Delta \vec{U}$ :

$$
\Delta \vec{U}=\left(U_{1}, U_{2} \cos \delta-U_{3} \sin \delta,-U_{2} \sin \delta-U_{3} \cos \delta\right)
$$

and the normal vector of the fault plane is $\vec{n}=(0,-\sin \delta,-\cos \delta)$.

The gravitational gradients are the 2 nd derivatives of the gravitational potential. Thus, gravitational gradient changes due to dislocation on a rectangular fault at a fixed point outside the half-space $(x \leq 0)$ can be calculated by applying various second order differential operators to the gravitational potential change:

Fig. 1 Point dislocation source buried in a half-space (Okubo 1992)

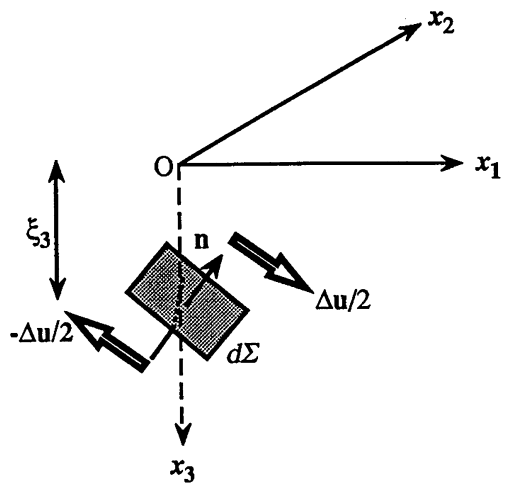


Fig. 2 Geometry of fault model (Okubo 1992)

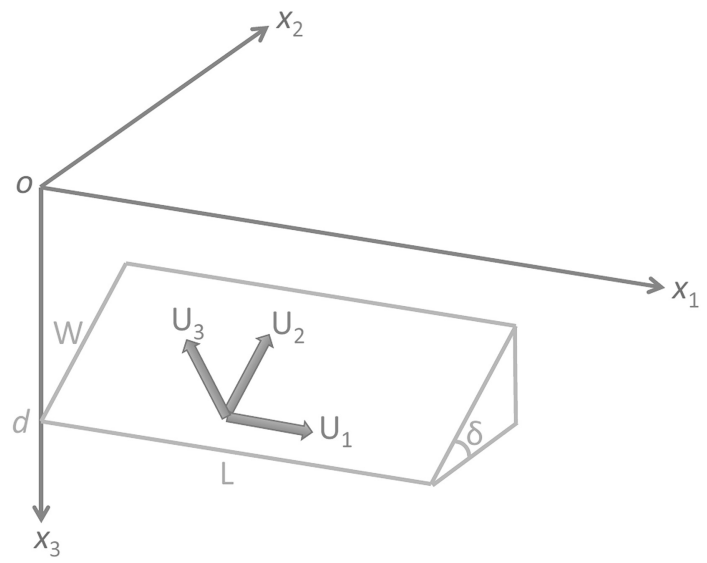

$$
\begin{gathered}
\Delta T_{i j}\left(x_{1}, x_{2}, 0\right)=-\frac{\partial^{2}}{\partial x_{i} \partial x_{j}} \Delta \Psi\left(x_{1}, x_{2}, 0\right)=-\left\{G \rho\left[U_{1} S_{i j}(\xi, \eta)+U_{2} D_{i j}(\xi, \eta)+U_{3} T_{i j}(\xi, \eta)\right]\right. \\
\left.+G \Delta \rho U_{3} C_{i j}(\xi, \eta)\right\} \quad(i, j=1,2,3) \\
\left(S_{i j}, D_{i j}, T_{i j}, C_{i j}\right)=\frac{\partial^{2}}{\partial x_{i} \partial x_{j}}(S, D, T, C)
\end{gathered}
$$

where $\Delta \Psi$ is a total potential change, $G$ is the gravitational constant, $S, D$ and $T$ are terms related to the potential changes caused by $U_{1}, U_{2}$ and $U_{3}$ dislocation components respectively, and $C$ takes into account the attraction of the mass intruding into the cavity formed by tensile opening.

The expressions of these components are listed in Appendix 1.

\subsection{Gravitational gradient changes using GRACE coefficients}

The geopotential coefficients of monthly gravity data of Level-2 which are consist of complete sets of spherical harmonic (Stokes) coefficients computed by Center Space Research (CSR05) have been used in this research. These coefficients have been fully normalized to degree and order 60, corresponding to the spatial resolution of $300 \mathrm{~km}$ and above (Tapley et al. 2004). A total of 45 monthly gravitational field solutions covering the time period from 2008 January to 2011 December are used. The Stokes coefficients $C_{n m}$, $S_{n m}$ of each monthly field are then used to compute monthly gravitational gradients in spherical coordinate. Since the local gravitational gradient changes are of interest here, the local north-east-down (NED) frame at a point with spherical coordinates $(r, \theta, \lambda)$ is introduced: the $\mathrm{x}$-axis is directed to the north, the $\mathrm{y}$-axis to the east and the $\mathrm{z}$-axis downwards. The full gravitational gradient tensor (second derivatives of the gravitational potential) in this local NED frame is represented in Eshagh (2009) and Eshagh and Abdollahzadeh (2012).

Because of the correlated errors in the high-frequency components of GRACE data, 'destriping' and filtering techniques are commonly applied to obtain estimates of the timevariable signals. 
To remove this drawback, we use Gaussian filter with radius of $350 \mathrm{~km}$ (Wahr et al. 1998) which is necessary to reveal the fine pattern caused by the earthquake. Atmospheric pressure variations, ocean tides, and barotropic ocean signals have been removed by means of three models, the European Centre for Meteorological Weather Forecasting (ECMWF) model, the Finite Element Solution 2004 model (FES2004) (Lyard et al. 2006) and the MOG2D-G barotropic (Carrere and Lyard 2003) model respectively. To suppress the contamination from seasonal variations and extract the coseismic signals, we compute the difference of two 2-year mean gravitational gradient fields before and after the earthquakes (Wang et al. 2012b; Chen et al. 2007). The mean gravitational gradient field before the earthquakes is obtained by averaging 26 monthly solutions from 2008 January to 2010 February, and the mean field after the event is computed by taking the mean of 19 monthly solutions from 2010 March to 2011 December (the data of some months are not available), so that this mean field contains deformation signals from the earthquake. The coseismic gravity and gravitational gradient changes were then extracted by computing the difference between these two mean fields before and after the earthquake. In this way, the GRACE coefficients contain the coseismic signals associated with the 2010 Maule event.

It is emphasized that there are some uncertainties in our observational data. They emanates from a superposition of errors from different sources, whose separation is difficult. In situ hydrological and hydro-meteorological observations, under certain conditions, facilitate the identification of different uncertainty types, and the quantification of their contribution to the overall error budget (Riegger et al. 2012). Riegger et al. (2012) assessed monthly GRACE solutions, hydrological and hydro-meteorological data, and their respective combinations to ascertain their uncertainties. They identified, removed, and replaced Unphysical outliers in GRACE data which leads to a significant increase (up to $70 \%$ ) in correlation with hydrological and hydro-meteorological data, and also a sizeable reduction (up to $40 \%$ ) in noise level. On further statistical analysis, they identified physical signals that neither have hydrological nor hydro-meteorological character in the GRACE data at the residual level which they have a strong latitudinal dependence in particular equatorials region where our case study is not located.

\subsection{Sensitivity analysis}

Here, we analyze the sensitivity of our model to fault parameters, i.e., fault dip, depth, slip, strike, length and width. For this purpose, two artificial fault planes, which have strike of $90^{\circ}$ and different rake based on lateral or thrust Maule fault $\left(0^{\circ}\right.$ or $\left.90^{\circ}\right)$ is placed parallel to Chile trench. A rectangular fault plane with length $L=150 \mathrm{~km}$ and width $W=150 \mathrm{~km}$ is used similar to a violent earthquake with large fault which the top edge of the fault plane is fixed at a depth of $5 \mathrm{~km}$. Poisson's ratio is assumed to be 0.25 , and density of the medium is assumed to be $2670 \mathrm{~kg} / \mathrm{m}^{3}$, consistent with the average density of Earth's crust.

Wang et al. (2012b) have shown spectrum structure of the earthquake deformation and the related gravity and gravitational gradient changes. They plotted the percentage of the total signal energy as a function of spherical harmonic degree. They have shown that the total signal energy has a high value to a spherical harmonic degree 25 and after that degree, signal energy limits to the 0 so this means that its energy is to $150 \mathrm{~km}$ wave length so the fault length and width are allowed to take values of 10, 50, 100, 150 and $250 \mathrm{~km}$. For each length and width values, the seismic gravitational gradient changes are computed. Figure 3 shows the calculated changes for left-lateral and thrust fault. It can be seen that with increasing length and width to $100 \mathrm{~km}$, the gravitational gradient changes have a smooth 


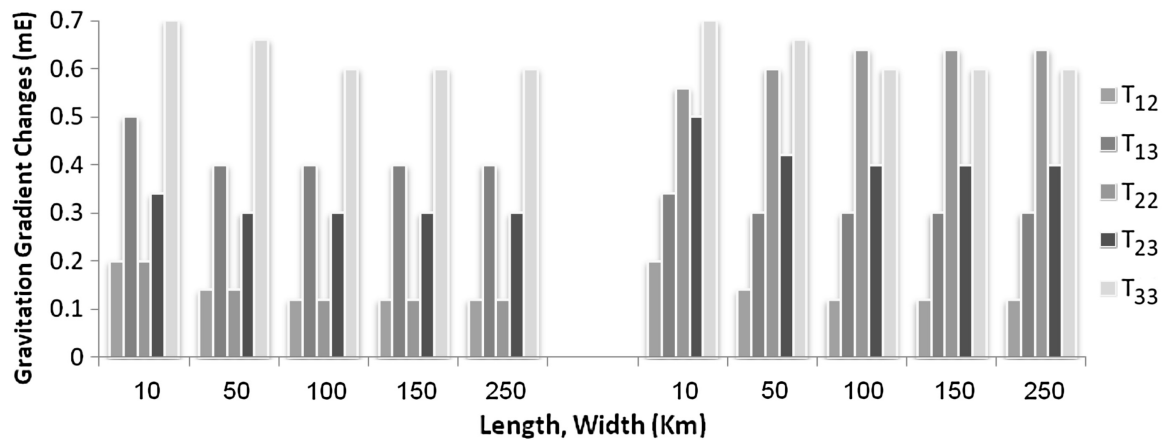

Fig. 3 The gravitational gradient changes which length and width of the fault plane varies from 10 to $250 \mathrm{~km}$ by left-lateral fault (left bar chart) and thrust fault (right bar chart) (in unit of $\mathrm{mE}$ )

trend and vary with length but from $100 \mathrm{~km}$ and above, the changes would be constant. This case is similar to all the components of gravitational gradient tensor, as well as leftlateral and thrust fault (Fig. 3).

In the next case, the fault slip is allowed to take values of 5, 10, 15, 20 and $25 \mathrm{~m}$. Figure 4 provides a map view to further illustrate the sensitivity of gravitational gradient changes for left-lateral and thrust fault slip. It can be seen that with increasing slip, the gravitational gradient changes have a linear trend and increase. This case is similar to all the components of gravitational gradient tensor, as well as left-lateral and thrust fault. As it is shown in this figure, fault slip creates a high effect on gravitational gradient changes so this parameter provides a constraint on the fault plane.

In other example, we test the model sensitivity to fault depth. The depth of the top edge of the fault varies from 5 to $35 \mathrm{~km}$. Figure 5 shows the calculated gravitational gradient changes for left-lateral and thrust fault depth. As could be seen in this figure, with increasing slip, the gravitational gradient changes have a non-linear trend and decrease. This case is similar to all the components of gravitational gradient tensor, as well as leftlateral and thrust fault.

In other case, the sensitivity of the model is tested to the fault strike. The strike angle of the fault plane takes values from 0 to $90^{\circ}$. Figure 6 shows the gravitational gradient changes for left-lateral and thrust fault similar to previous case with increasing strike angle.

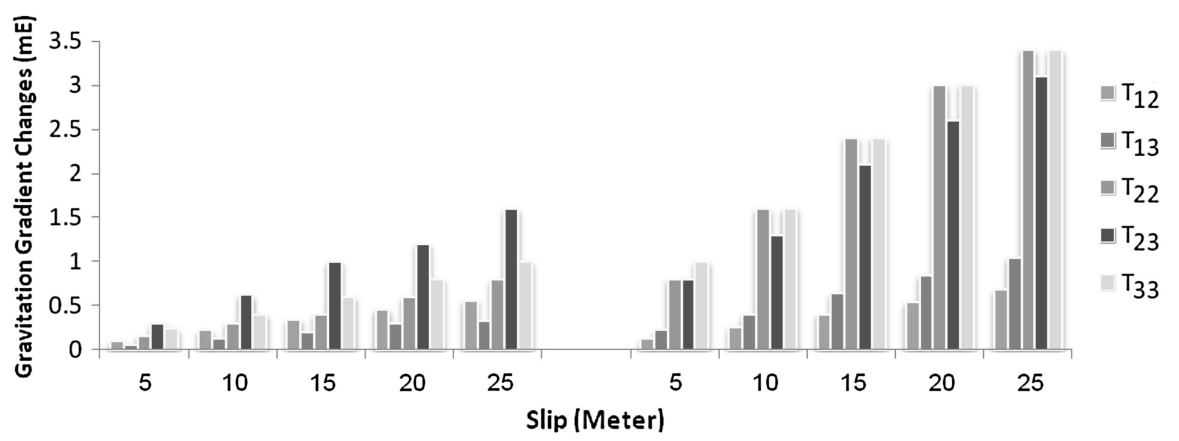

Fig. 4 The gravitational gradient changes which slip of the fault plane varies from 5 to $25 \mathrm{~m}$ by left-lateral fault (left bar chart) and thrust fault (right bar chart) (in unit of $\mathrm{mE}$ ) 


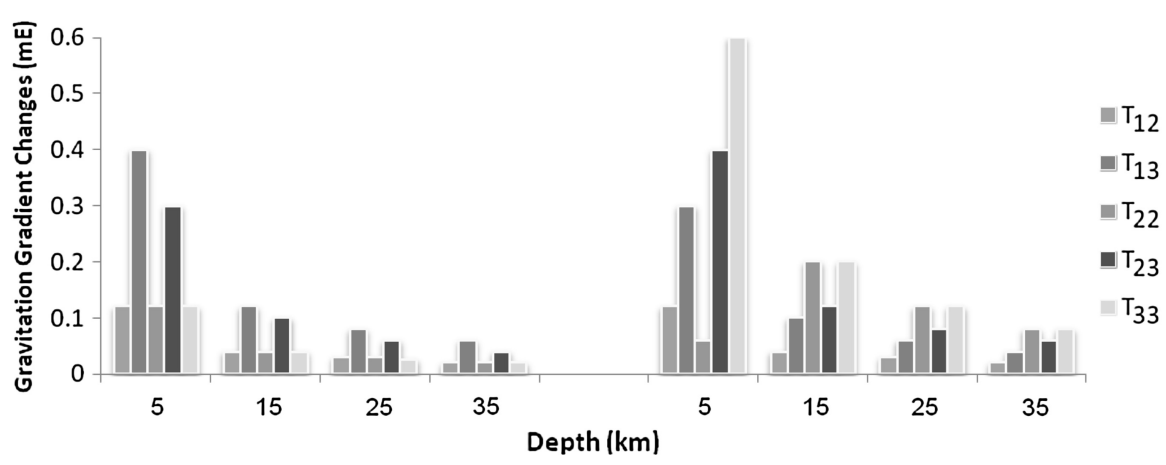

Fig. 5 The gravitational gradient changes which depth of the fault plane varies from $5 \mathrm{~km}$ to $35 \mathrm{~m}$ by leftlateral fault (left bar chart) and thrust fault (right bar chart) (in unit of $\mathrm{mE}$ )

As can be seen from Fig. 6, the gravitational gradient changes do not vary with increasing strike angle. This result is similar to left-lateral and thrust fault. As a result, the strike information from other models or observations should be used in order to invert desired model for other fault parameters.

In a last case, the fault dip is allowed to take values of $0,30,45,60$ and $90^{\circ}$. Figure 7 shows the gravitational gradient changes for left-lateral and thrust fault with increasing dip angle of the fault plane. As can be seen in this figure, contrary to the previous case, the behavior of the left-lateral and thrust fault is different with changing dip angle. Also the behavior of each components of the gravitational gradient tensor is different. Moreover, this parameter similar to the slip, has a high effect on gravitational gradient changes.

The result of the sensitivity analysis revealed that the model is sensitive to the most of the fault parameters such as slip, depth, dip and is not sensitive to the strike. Also the results show that length and width of fault to a certain level have sensitivity to the model. Also the behavior of each parameter except dip is similar for left-lateral and thrust fault. Moreover, the behavior of each of the components of gravitational gradient tensor is similar except for dip parameter. Moreover changing the dip and slip parameters have a more influence on gravitational gradient change in comparison with other parameters.

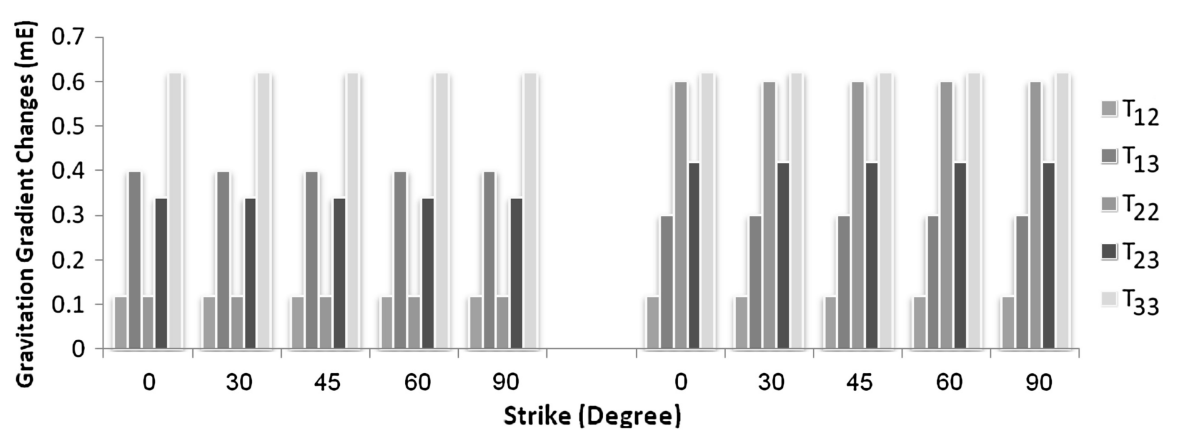

Fig. 6 The gravitational gradient changes which strike of the fault plane varies from 0 to $90^{\circ}$ by left-lateral fault (left bar chart) and thrust fault (right bar chart) (in unit of $\mathrm{mE}$ ) 


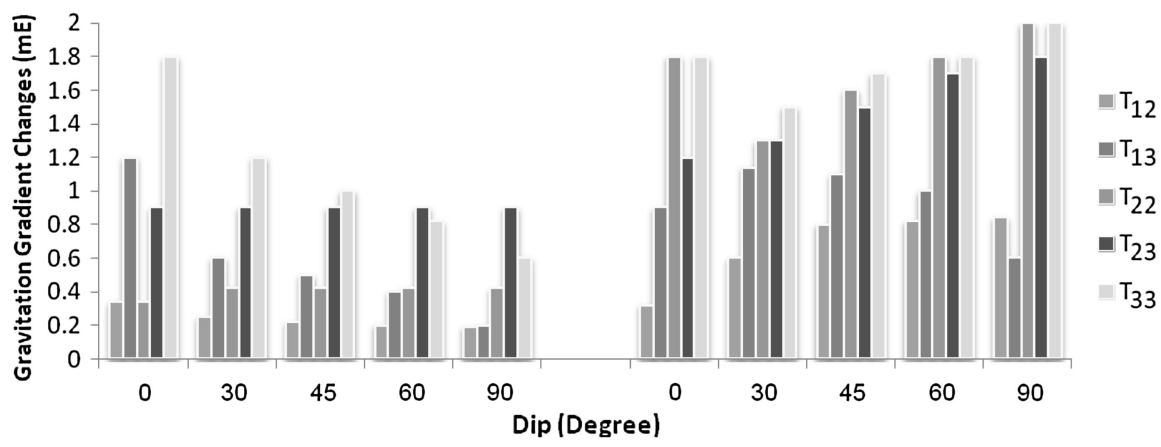

Fig. 7 The gravitational gradient changes which dip of the fault plane varies from 0 to $90^{\circ}$ by left-lateral fault (left bar chart) and thrust fault (right bar chart) (in unit of $\mathrm{mE}$ )

\section{Numerical results and case study}

The Saturday, February 27, 2010 at 06:34:14 UTC Maule Chilean earthquake, which was caused by the subduction of the Nazca plate underneath the overlying South America plate, is the sixth largest event in the seismic record. The Mw 8.8 Maule earthquake permanently changed the mass distribution within the Earth and consequently its gravitational potential, which can be observed with temporal and spatial resolutions of a month and several

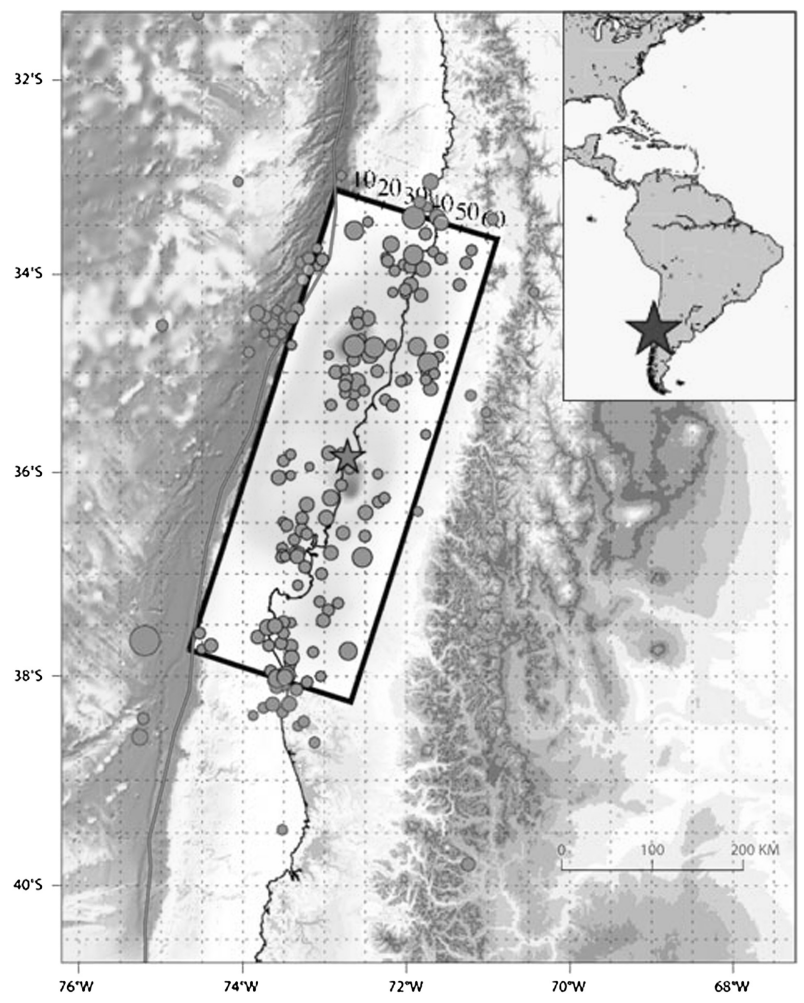

Fig. 8 Surface projection of the slip distribution superimposed on Maule (USGS) 
hundred km, respectively, using data from the GRACE satellite mission (Wang et al. 2012a) so the finite fault model for the Maule earthquake are used to predict the coseismic gravity and gravitational gradient changes. Figure 8 shows the fault planes in Maule.

Figure 9 shows time series of three components of gravitational gradient changes $\left(T_{22}, T_{33}\right.$ and $\left.T_{23}\right)$ from GRACE observations when the concentration centers (earthquake centers) are located at $\left(\varphi=-35.5^{\circ}\right.$ and $\left.\lambda=-72.5^{\circ}\right)$ and $\left(\varphi=-35.5^{\circ}\right.$ and $\left.\lambda=-69.5^{\circ}\right)$ respectively for expansion and contraction region (Fig. 10). These time series are applied for two sides of fault plane from January 2003 to July 2014.

Those gravitational gradient changes that are obtained in March 2010, are illustrated after correction of seasonal effects to extract the coseismic signals. As can be seen in these figures, it is clear that these positive and negative changes (it means there are extension and contraction area in fault region which have been due to increased or decreased density) are corresponding to the earthquake signals. Moreover, the significant gravitational gradient variations (about -1.04 and $1.02 \mathrm{mE}$ ) have been occurred in two opposite sides of the fault plane after the earthquake (step function is shown with red line) and could be the result of rapture on corresponding fault segments.
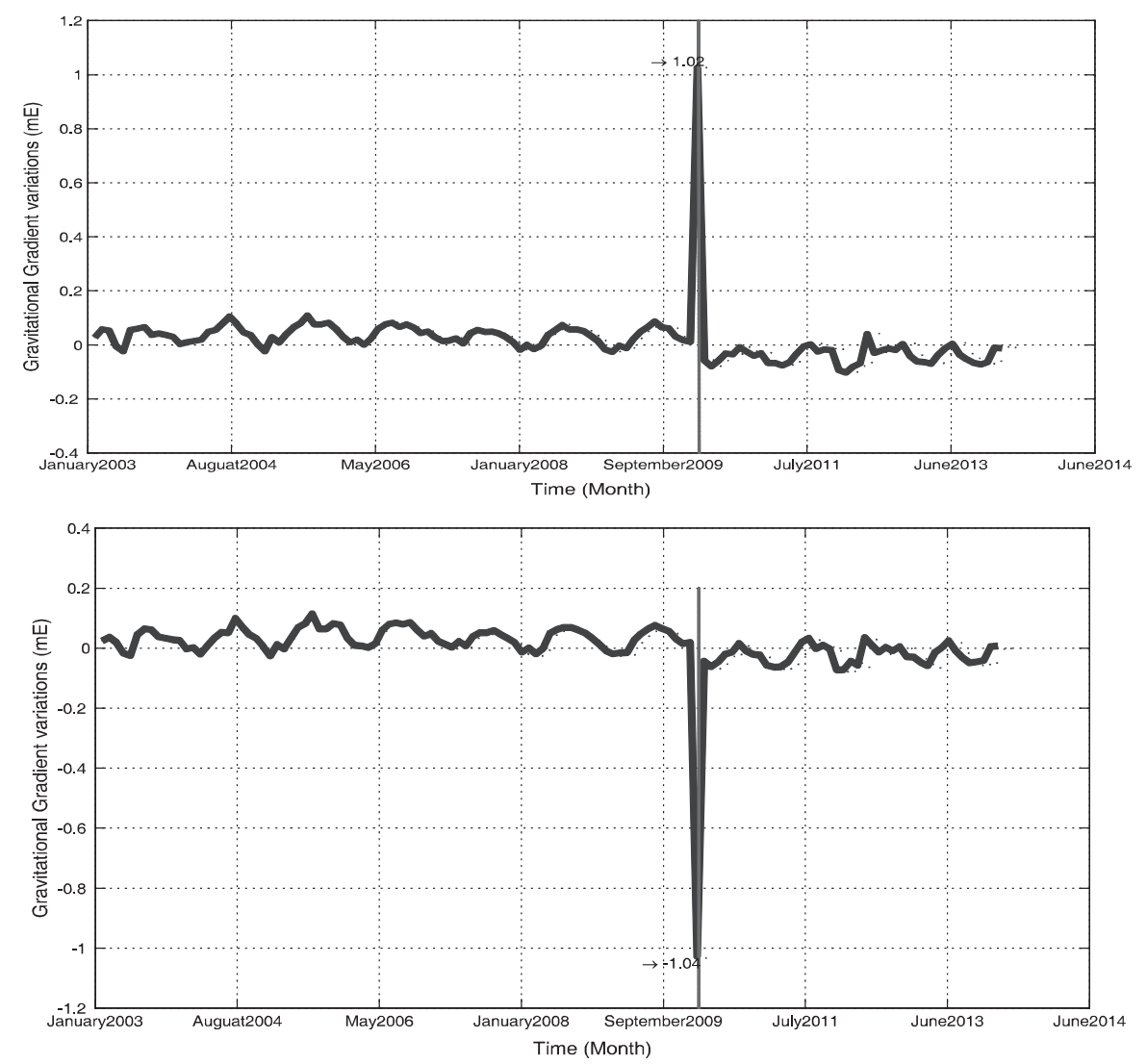

Fig. 9 Time series of gravitational gradient variations from January 2003 to July 2014 after correction of seasonal effects in March 2010 at a point with $\varphi=-35.5^{\circ}$ and $\lambda=-72.5^{\circ}$ (top figure) and $\varphi=-35.5^{\circ}$ and $\lambda=-69.5^{\circ}$ (down figure) 

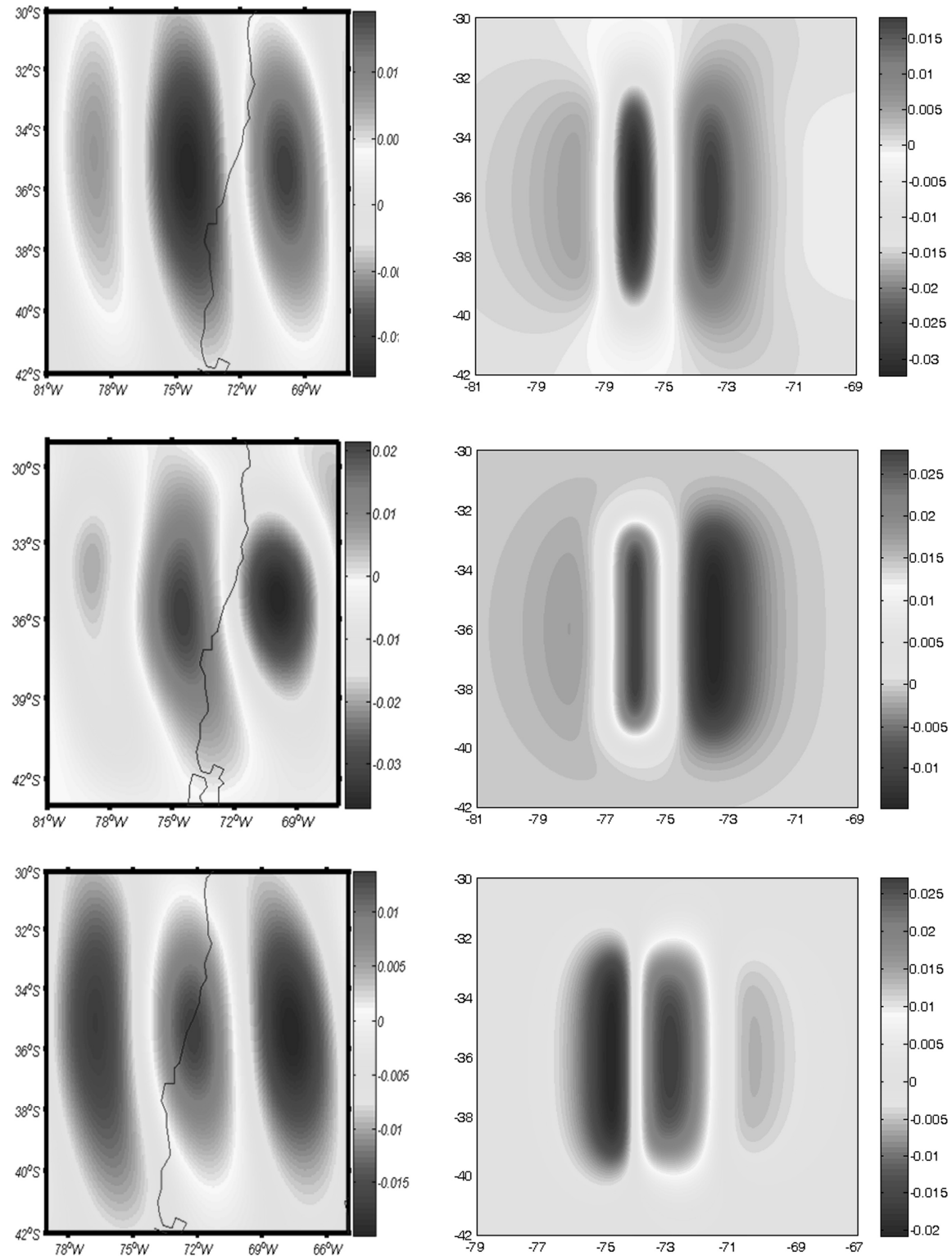

Fig. 10 Gravitational gradient changes by GRACE (left column), analytical model (right column) (in unit of $\mathrm{mE}$ )

It is necessary to mention that the coordinate systems for derivation of gravity and gravity gradient change from the analytical model and GRACE observations are not similar to each other. Actually, their x-axis has rotated by the strike angle. This angle are valuated as an unknown fault parameter by comparing the gravity change in two coordinate system (Fig. 10) In order to estimate this angle, the genetic algorithm is used in this research. We can use a nonlinear model as: 


$$
L=F(\delta)
$$

in which $L$ are observations and $F$ is a function of $\delta$ which is strike angle of the fault. The selected criterion for measuring chromosome in genetic algorithm is chosen as:

$$
R_{i}=\left\|\vec{L}_{i}-\vec{F}\left(\delta_{i}\right)\right\|
$$

in which $R_{i}$ is residual norm between observations and model. The initial fault parameters for proposed model in adopted from data of USGS. The result shows that the strike angle which is the rotation between two systems is about $13^{\circ}$.

Here we compute the gravitational gradient changes and use it to discuss about the coseismic deformation resulting from the Maule earthquake. Figure 10 shows the gravitational gradient change components such as $T_{22}, T_{23}$, and $T_{33}$ following the 2010 Maule earthquake using GRACE (spherical harmonic coefficients) and analytical model (fault parameters). The peak value in the negative signal on the land from GRACE observations is about $0.03 \mathrm{mE}$ and the maximum positive signal is about $0.01 \mathrm{mE}$ in the ocean. Similarly, the peak value in the negative signal on the land from analytical model is about $0.04 \mathrm{mE}$ and the maximum positive signal is about $0.01 \mathrm{mE}$ in the ocean. The positive signal in the ocean due to sea-floor uplift is only about $0.01 \mathrm{mE}$ at GRACE spatial resolution; this is close to the GRACE error level. Due to the overall of these uncertainties of gravitational gradient changes, we state that the GRACE observations shown in Fig. 10 resolve almost all the negative signals on land caused by the Maule earthquake, while the detected positive signals over the ocean qualitatively correspond to seafloor uplift, but should not be quantitatively applied in the inversion. Since the high-frequency contents in gravitational field variation can be amplified by deriving the gravitational gradients, the GRACE-derived co-seismic gravitational gradient changes clearly delineate the fault line, locate significant slips, better define the extent of the co-seismic deformation and reveal refined mass redistribution features caused by the earthquake (Wang et al. 2012b).

As is shown in the above figures, gravitational gradient changes for $T_{22}, T_{33}$ and $T_{23}$ components have computed 0.03, 0.06 and $0.04 \mathrm{mE}$ respectively using GRACE observations, also these variations using analytical model have computed 0.05, 0.05 and $0.06 \mathrm{mE}$ respectively that shows there is good accordance between the results of two approaches. The discrepancy observed in values of these results may be explained by the difference between considered surfaces in these approaches, because the results with GRACE have related to the actual Earth which has a rough topography while the results of analytical model assume the plane shape of Earth's surface.

In order to investigate whether GRACE observations can provide constraints for fault inversion, a further analyze with a nonlinear inversion technique is applied (see Eq 4 which is used for strike parameter). In this case, the genetic algorithm is used to simultaneously invert for the fault parameters such as depth, dip and average slip using gravitational gradient changes observable from GRACE. In order to further investigate parameter uncertainties induced by GRACE observation errors, we also use the lower and upper bounds of a posteriori error estimates for the GRACE observations to invert for fault-plane depth, dip and average slip. The ultimate optimal estimate for fault depth, dip and slip are $24.6 \mathrm{~km}, 15^{\circ}$, and $8 \mathrm{~m}$ respectively. Our results have compared with existing slip models obtained by various constraints or via inversion of observations, including uplifted/subsided biomarkers, teleseismic data, InSAR, GPS, and tsunami observations. Comparison has shown good accordance so we conclude that the GRACE-derived amplitude can be 
used to constrain the fault parameters of the Maule earthquake, and these are not discernibly different from the amplitudes derived by analytical model.

\section{Conclusion}

In this study the gravitational gradient changes caused by faulting on a finite rectangular plane buried in a homogenous half-space have been computed using an analytical model which is a function of fault parameters. Also the components of this tensor have been determined for faults with different dip angles. The results have been showed the amplitude of gravitation and gravitational gradient changes for a thrust fault is more than strike-slip fault. These results did not change with increasing the dip angle of the fault. Also the $T_{33}$ component has the most gravitational gradient changes among the other components. Moreover, gravity and gravitational gradient changes using GRACE observations have been computed for 2010 Chile, Maule earthquake without any required information about fault parameters. The results have been compared to analytical geodynamic model. Gravitational gradient changes for $T_{22}, T_{33}$ and $T_{23}$ components have been computed 0.03 , 0.06 and $0.04 \mathrm{mE}$ respectively using GRACE observations, also these variations using analytical model have been computed $0.05,0.05$ and $0.06 \mathrm{mE}$ respectively which demonstrate a good agreement between GRACE observations and analytical models in computed gravitation and gravitational gradient changes.

\section{Appendix 1: Gravitational gradient change caused by fault}

$$
\begin{aligned}
S_{12}(\xi, \eta)= & -\sin \delta \sec ^{2} \delta \frac{\partial I_{0}}{\partial x_{1}}-\left(q \sec \delta-x_{3}\right) \sec \delta \frac{\partial^{2} I_{0}}{\partial x_{1} \partial x_{2}}+2 \tan ^{2} \delta \frac{\partial I_{1}}{\partial x_{2}}+2 \xi \tan ^{2} \delta \frac{\partial^{2} I_{1}}{\partial x_{1} \partial x_{2}} \\
& -\frac{\bar{y} \xi \tan \delta}{R^{3}}
\end{aligned}
$$

$$
S_{13}(\xi, \eta)=-\frac{\xi q \sin \delta}{R^{3}}+\frac{\xi q^{2}(2 R+\eta) \cos \delta}{R^{3}(R+\eta)^{2}}
$$$$
S_{22}(\xi, \eta)=\left(\frac{1}{R}-\frac{\bar{y}^{2}}{R^{3}}\right) \tan \delta-2 \frac{\partial I_{0}}{\partial x_{2}} \sin \delta \sec ^{2} \delta-q \frac{\partial^{2} I_{0}}{\partial x_{2}^{2}} \sec ^{2} \delta+2 \xi \frac{\partial^{2} I_{1}}{\partial x_{2}^{2}} \tan ^{2} \delta
$$

$$
S_{23}(\xi, \eta)=\frac{\sin ^{2} \delta}{R}-\frac{2 q \sin \delta \cos \delta}{R(R+\eta)}-\frac{\bar{y} q \sin \delta}{R^{3}}+\frac{\bar{y} q^{2}(2 R+\eta) \cos \delta+R^{2} q^{2} \cos ^{2} \delta}{R^{3}(R+\eta)^{2}}
$$

$$
\begin{aligned}
S_{33}(\xi, \eta)= & \frac{\sin \delta \cos \delta}{R}+\frac{q\left(\sin ^{2} \delta-\cos ^{2} \delta\right)}{R(R+\eta)}+\frac{\bar{d} q \sin \delta}{R^{3}} \\
& -\frac{\bar{d} q^{2}(2 R+\eta) \cos \delta+R^{2} q^{2} \sin \delta \cos \delta}{R^{3}(R+\eta)^{2}}
\end{aligned}
$$

$$
D_{12}(\xi, \eta)=-\tan \delta \frac{\partial I_{0}}{\partial x_{2}}-\xi \tan \delta \frac{\partial^{2} I_{0}}{\partial x_{1} \partial x_{2}}-2 \sin \delta \tan \delta \frac{\partial I_{1}}{\partial x_{1}}-2 q \tan \delta \frac{\partial^{2} I_{1}}{\partial x_{1} \partial x_{2}}-\frac{\sin \delta}{R}+\frac{\bar{y} q}{R^{3}}
$$




$$
\begin{aligned}
& D_{13}(\xi, \eta)=-\frac{q \bar{d}}{R^{3}}-\frac{q \sin \delta}{R(R+\eta)} \\
& D_{22}(\xi, \eta)=-\frac{2 \bar{y} \sin \delta+q}{R(R+\xi)}+\frac{\bar{y}^{2} q(2 R+\xi)}{R^{3}(R+\xi)^{2}}-\xi \frac{\partial^{2} I_{0}}{\partial x_{2}^{2}} \tan \delta-4 \frac{\partial I_{1}}{\partial x_{2}} \sin \delta \tan \delta-2 q \frac{\partial^{2} I_{1}}{\partial x_{2}^{2}} \tan \delta \\
& D_{23}(\xi, \eta)=\frac{2 \bar{d} \sin \delta}{R(R+\xi)}+\frac{\xi \sin ^{2} \delta}{R(R+\eta)}-\frac{q \bar{d} \bar{y}(2 R+\xi)}{R^{3}(R+\xi)^{2}} \\
& D_{33}(\xi, \eta)=\frac{\bar{y} \sin \delta+\bar{d} \cos \delta}{R(R+\xi)}+\frac{\xi \sin \delta \cos \delta}{R(R+\eta)}+\frac{q \bar{d}^{2}(2 R+\xi)}{R^{3}(R+\xi)^{2}} \\
& T_{12}(\xi, \eta)=\tan ^{2} \delta\left(\frac{\partial I_{0}}{\partial x_{2}}+\xi \frac{\partial^{2} I_{0}}{\partial x_{1} \partial x_{2}}\right)+2 \tan ^{2} \delta\left(\sin \delta \frac{\partial I_{1}}{\partial x_{1}}+q \frac{\partial^{2} I_{1}}{\partial x_{1} \partial x_{2}}\right) \\
& +2\left(\sin \delta \frac{\partial I_{2}}{\partial x_{1}}+q \frac{\partial^{2} I_{2}}{\partial x_{1} \partial x_{2}}\right)+C_{12}(\xi, \eta) \\
& T_{13}(\xi, \eta)=\frac{q \bar{y}}{R^{3}}-\frac{2 q \cos \delta}{R(R+\eta)}+\frac{q \xi^{2}(2 R+\eta) \cos \delta}{R^{3}(R+\eta)^{2}} \\
& T_{22}(\xi, \eta)=\xi \frac{\partial^{2} I_{0}}{\partial x_{2}^{2}} \tan ^{2} \delta+2\left(2 \frac{\partial I_{1}}{\partial x_{2}} \sin \delta+q \frac{\partial^{2} I_{1}}{\partial x_{2}^{2}}\right) \tan ^{2} \delta+2\left(2 \frac{\partial I_{2}}{\partial x_{2}} \sin \delta+q \frac{\partial^{2} I_{2}}{\partial x_{2}^{2}}\right) \\
& +C_{22}(\xi, \eta) \\
& T_{23}(\xi, \eta)=-\frac{2 q}{R(R+\xi)}+\frac{q \bar{y}^{2}(2 R+\xi)}{R^{3}(R+\xi)^{2}}+\frac{\xi q \bar{y}(2 R+\eta) \cos \delta+\xi q R^{2} \cos ^{2} \delta}{R^{3}(R+\eta)^{2}} \\
& T_{33}(\xi, \eta)=\frac{\eta}{R(R+\xi)}+\frac{\xi}{R(R+\eta)}-\frac{q \bar{d} \bar{y}(2 R+\xi)}{R^{3}(R+\xi)^{2}}-\frac{\xi q\left[\bar{d}(2 R+\eta)+R^{2} \sin \delta\right] \cos \delta}{R^{3}(R+\eta)^{2}} \\
& C_{12}(\xi, \eta)=-\frac{\cos \delta}{R}-\frac{q \sin \delta}{R(R+\eta)} \\
& C_{13}(\xi, \eta)=\frac{\sin \delta}{R}-\frac{q \cos \delta}{R(R+\eta)} \\
& C_{22}(\xi, \eta)=\frac{2 \bar{d} \sin \delta-\eta}{R(R+\xi)}+\frac{\xi \sin ^{2} \delta}{R(R+\eta)} \\
& C_{23}(\xi, \eta)=\frac{\bar{y} \sin \delta+\bar{d} \cos \delta}{R(R+\xi)}+\frac{\xi \sin \delta \cos \delta}{R(R+\eta)}
\end{aligned}
$$




$$
\begin{aligned}
& C_{33}(\xi, \eta)=-\frac{2 \bar{d} \sin \delta-\eta}{R(R+\xi)}+\frac{\xi \cos ^{2} \delta}{R(R+\eta)} \\
& \frac{\partial I_{0}}{\partial x_{1}}=\frac{\xi}{R}\left(\frac{1}{R+\eta}-\frac{\sin \delta}{R+\bar{d}}\right) \\
& \frac{\partial I_{0}}{\partial x_{2}}=\frac{\bar{y}+R \cos \delta}{R(R+\eta)}-\frac{\bar{y} \sin \delta}{R(R+\bar{d})} \\
& \frac{\partial^{2} I_{0}}{\partial x_{1} \partial x_{2}}=-\frac{\xi(R \cos \delta-q)(2 R+\bar{d})}{R^{3}(R+\bar{d})^{2}}+\frac{\xi \cos \delta}{R^{2}(R+\bar{d})}-\frac{q \xi(2 R+\eta) \sin \delta}{R^{3}(R+\eta)^{2}} \\
& \frac{\partial^{2} I_{0}}{\partial x_{2}^{2}}=\frac{q \bar{y}(2 R+\bar{d})-R^{2}(R \sin \delta+\eta)}{R^{3}(R+\bar{d})^{2}}-\frac{q \bar{y}(2 R+\eta) \sin \delta-R^{2}(R \sin \delta+\bar{d}) \sin \delta}{R^{3}(R+\eta)^{2}} \\
& \frac{\partial I_{1}}{\partial x_{1}}=-\frac{\eta \cos \delta}{2 R(R+\bar{d})}-\frac{q^{2} \cos \delta}{2 R(R+\eta)(R+\bar{d})}+\frac{q(1-\sin \delta)}{2(R+\eta)(R+\bar{d})} \\
& \frac{\partial I_{1}}{\partial x_{2}}=\frac{\xi}{2 R(R+\bar{d})}-\frac{\xi \sin \delta}{2 R(R+\eta)} \\
& \frac{\partial^{2} I_{1}}{\partial x_{1} \partial x_{2}}=\frac{1}{2 R(R+\bar{d})}-\frac{\xi^{2}(2 R+\bar{d})}{2 R^{3}(R+\bar{d})^{2}}-\frac{\sin \delta}{2 R(R+\eta)}+\frac{\xi^{2}(2 R+\eta) \sin \delta}{2 R^{3}(R+\eta)^{2}} \\
& \frac{\partial^{2} I_{1}}{\partial x_{2}^{2}}=\frac{\xi\left(\bar{y}(2 R+\eta)+R^{2} \cos \delta\right) \sin \delta}{2 R^{3}(R+\eta)^{2}}-\frac{\xi \bar{y}(2 R+\bar{d})}{2 R^{3}(R+\bar{d})^{2}} \\
& \frac{\partial I_{2}}{\partial x_{1}}=\frac{q}{2 R(R+\eta)} \\
& \frac{\partial I_{2}}{\partial x_{2}}=-\frac{\bar{d}}{2 R(R+\xi)}-\frac{\xi \sin \delta}{2 R(R+\eta)} \\
& \frac{\partial^{2} I_{2}}{\partial x_{1} \partial x_{2}}=\frac{\bar{d}}{2 R^{3}}-\frac{\sin \delta}{2 R(R+\eta)}+\frac{\xi^{2}(2 R+\eta) \sin \delta}{2 R^{3}(R+\eta)^{2}} \\
& \frac{\partial^{2} I_{2}}{\partial x_{2}^{2}}=\frac{\bar{d} \bar{y}(2 R+\xi)}{2 R^{3}(R+\xi)^{2}}+\frac{\xi \sin \delta\left(\bar{y}(2 R+\eta)+R^{2} \cos \delta\right)}{2 R^{3}(R+\eta)^{2}}
\end{aligned}
$$

if $\cos \delta=0$

$$
\begin{aligned}
S_{12}(\xi, \eta)= & \frac{\xi \sin ^{2} \delta}{2 R(R+\bar{d})}-\frac{(\eta \sin \delta+q \cos \delta) \xi \sin ^{2} \delta}{2 R(R+\bar{d})^{2}}-\frac{\xi(R \sin \delta+q \cos \delta) \sin ^{2} \delta}{R^{2}(R+\eta)} \\
& +\frac{\xi q(\bar{y}+R \cos \delta)(2 R+\eta) \sin ^{2} \delta}{R^{2}(R+\eta)^{2}}-\frac{\xi q \bar{y}(2 R+\bar{d}) \sin \delta}{2 R^{3}(R+\bar{d})^{2}}+\frac{\xi q \eta \bar{y}(3 R+\bar{d}) \sin ^{2} \delta}{2 R^{3}(R+\bar{d})^{3}}
\end{aligned}
$$




$$
\begin{gathered}
D_{12}(\xi, \eta)=-\frac{\sin \delta}{R}+\frac{q \bar{y}}{R^{3}} \\
T_{12}(\xi, \eta)=2 \frac{\partial I_{2}}{\partial x_{1}} \sin \delta+2 q \frac{\partial^{2} I_{2}}{\partial x_{1} \partial x_{2}}+\frac{R \cos \delta+\bar{y} \sin \delta}{2 R(R+\bar{d})}-\left(\frac{\xi^{2} \cos \delta+\eta \bar{y}}{2 R(R+\bar{d})^{2}}\right) \\
-\frac{\xi^{2} \bar{y}(2 R+\bar{d}) \sin \delta}{2 R^{3}(R+\bar{d})^{2}}+\frac{\xi^{2} \eta \bar{y}(3 R+\bar{d})}{2 R^{3}(R+\bar{d})^{3}} \\
S_{22}(\xi, \eta)=-\frac{(2 \bar{y} \sin \delta+q) \sin ^{2} \delta}{R(R+\eta)}+\frac{\bar{y} \sin ^{2} \delta}{2 R(R+\bar{d})}-\frac{\left(R \eta \sin ^{2} \delta+q \bar{y}\right) \bar{y} \sin \delta}{2 R^{2}(R+\bar{d})^{2}} \\
-\frac{(\eta \sin \delta-R-\bar{d}) \sin \delta}{2 R(R+\bar{d})^{2}}\left(\bar{y} \sin \delta+q-\frac{q \bar{y}^{2}(3 R+\bar{d})}{R^{2}(R+\bar{d})}\right)+\frac{q \bar{y}^{2}(2 R+\eta) \sin ^{2} \delta}{R^{3}(R+\eta)^{2}} \\
D_{22}(\xi, \eta)=-\frac{2 \bar{y} \sin \delta+q}{R(R+\xi)}+\frac{q \bar{y}^{2}(2 R+\xi)}{R^{3}(R+\xi)^{2}} \\
T_{22}(\xi, \eta)=\frac{\partial^{2} C}{\partial x^{2}}+2 \frac{\partial I_{2}}{\partial x_{2}} \sin \delta+2\left(q+\sin ^{2} \frac{\partial^{2} I_{2}}{\partial x_{2}^{2}}+\frac{\xi R \sin \delta}{2 R^{2}(R+\bar{d})}\right. \\
-\frac{\xi \eta}{2 R(R+\bar{d})^{2}}-\frac{\xi \bar{y}^{2}(2 R+\bar{d}) \sin _{2}}{2 R^{3}(R+\bar{d})^{2}}+\frac{\xi \eta \bar{y}^{2}(3 R+\bar{d})}{2 R^{3}(R+\bar{d})^{3}}
\end{gathered}
$$

\section{References}

Carrere L, Lyard F (2003) Modeling the barotropic response of the global ocean to atmospheric wind and pressure forcing comparisons with observations. Geophys Res Lett 30(6):1275. doi:10.1029/ 2002GL016473

Chen JL, Wilson CR, Tapley BD, Grand S (2007) GRACE detects coseismic and postseismic deformation from the Sumatra-Andaman earthquake. Geophys Res Lett 34(13):doi:10.1029/2007GL030356

De Linage C, Rivera L, Hinderer J, Boy JP, Rogister Y, Lambotte S, Biancale R (2009) Separation of coseismic and postseismic gravity changes for the 2004 Sumatran earthquake from $4.6 \mathrm{yr}$ of GRACE observations and modelling of the coseismic change by normal mode summation. Geophys $\mathrm{J}$ Int 176:695-714. doi:10.1111/j.1365-246X.2008.04025.x

Eshagh M (2009) Alternative expressions for gravity gradients in local north-oriented frame and tensor spherical harmonics. Acta Geophys 58:215-243. doi:10.2478/s11600-009-0048-z

Eshagh M, Abdollahzadeh M (2012) Software for generating gravity gradients using a geopotential model based on an irregular semi vectorization algorithm. Com Geosci 39:152-160. doi:10.1016/j.cageo. 2011.06.003

Han SC, Sauber J, Luthcke S (2010) Regional gravity decrease after the 2010 Maule (Chile) earthquake indicates large-scale mass redistribution. Geophys Res Lett 37:L23307. doi:10.1029/2010GL045449

Han SC, Riva R, Sauber J, Okal E (2013) Source parameter inversion for recent great earthquakes from a decade-long observation of global gravity fields. J Geophys Res 118:1240-1267. doi:10.1002/jgrb. 50116

Heki K, Matsuo K (2010) Coseismic gravity changes of the 2010 earthquake in central Chile from satellite gravimetry. Geophys Res Lett 37:L24306. doi:10.1029/2010GL045335

Lyard F, Lefevre F, Letellier T, Francis O (2006) Modelling the global ocean tides: modern insights from FES2004. Ocean Dyn 56:394-415. doi:10.1007/s10236-006-0086-X

Maruyama T (1964) Statical elastic dislocation in an infinite and semi-infinite medium. Bull Earthquake Res Inst Tokyo Univ 42:289-368 
Ogawa R, Heki K (2007) Slow postseismic recovery of geoid depression formed by the 2004 SumatraAndaman Earthquake by mantle water diffusion. Geophys Res Lett 34:L06313. doi:10.1029/ 2007GL029340

Okada Y (1985) Surface deformation due to shear and tensile faults in a half-space. Bull Seism Soc Am $75: 1135-1154$

Okubo S (1991) Potential and gravity changes raised by point dislocations. Geophys J Int 105:573-586

Okubo S (1992) Gravity and potential changes due to shear and tensile faults in a halfspace. J Geophys Res 97(B5):7137-7144

Riegger J, Tourian MJ, Devaraju B, Sneeuw N (2012) Analysis of GRACE uncertainties by hydrological and hydro-meteorological observations. J Geodyn 59:16-27. doi:10.1016/j.jog.2012.02.001

Steketee JA (1958) On Volterra's dislocations in a semi-infinite elastic medium. Can J Phys 36:192-205

Tapley BD, Bettadpur S, Watkins M, Reigber C (2004) The gravity recovery and climate experiment: mission overview and early results. Geophys Res Lett 31:L09607. doi:10.1029/2004GL019920

Wahr J, Molenaar M, Bryan F (1998) Time variability of the Earth's gravity field: hydrological and oceanic effects and their possible detection using GRACE. J Geophys Res 103:30229-32205. doi:10.1029/ 98JB02844

Wang L, Shum CK, Simons FJ, Tassara A, Erkan K, Jekeli C, Braun A, Kuo CY, Lee H, Yuan DY (2012a) Coseismic slip of the $2010 \mathrm{Mw} 8.8$ Great Maule, Chile earthquake quantified by GRACE observation. Earth Planet Sci Lett 335:167-179. doi:10.1016/j.epsl.2012.04.044

Wang L, Shum CK, Jekeli C (2012b) Gravitational gradient changes following the Summatra-Andaman earthquake inferred from GRACE. Geophys J Int 191:1109-1118. doi:10.1111/j.1365-246X.2012. 05674.x 